\title{
Cerebrocerebellar Hypometabolism Associated With Repetitive Blast Exposure Mild Traumatic Brain Injury in 12 Iraq War Veterans With Persistent Post-Concussive Symptoms
}

\author{
Elaine R. Peskind, MD ${ }^{\star}, 1,3$, Eric C. Petrie, MD ${ }^{1,3}$, Donna J. Cross, PhD $^{4}$, Kathleen \\ Pagulayan, PhD 1,3 , Kathleen McCraw, BA ${ }^{1}$, David Hoff, PA-C ${ }^{1}$, Kim Hart, PA-C ${ }^{1}$, Chang-En \\ Yu, PhD ${ }^{2,5}$, Murray A. Raskind, MD ${ }^{1,3}$, David G. Cook, PhD ${ }^{2,5}$, and Satoshi Minoshima, MD, \\ $\mathrm{PhD}^{4}$ \\ ${ }^{1}$ Northwest Network Mental Illness, Research, Education, and Clinical Center (MIRECC), \\ Veterans Affairs Puget Sound Health Care System, Seattle, WA, USA \\ ${ }^{2}$ Geriatric Research, Education, and Clinical Center (GRECC), Veterans Affairs Puget Sound \\ Health Care System, Seattle, WA, USA \\ ${ }^{3}$ Department of Psychiatry and Behavioral Sciences, University of Washington, Seattle, WA, USA \\ ${ }^{4}$ Department of Radiology, University of Washington, Seattle, WA, USA \\ ${ }^{5}$ Department of Medicine, University of Washington, Seattle, WA, USA
}

\begin{abstract}
Disagreement exists regarding the extent to which persistent post-concussive symptoms (PCS) reported by Iraq combat Veterans with repeated episodes of mild traumatic brain injury (mTBI) from explosive blasts represent structural or functional brain damage or an epiphenomenon of comorbid depression or posttraumatic stress disorder (PTSD). Objective assessment of brain function in this population may clarify the issue. To this end, twelve Iraq war Veterans ( $32.0 \pm 8.5$ [mean \pm standard deviation (SD)] years of age) reporting one or more blast exposures meeting American College of Rehabilitation Medicine criteria for mTBI and persistent PCS and 12 cognitively normal community volunteers $(53.0 \pm 4.6$ years of age $)$ without history of head trauma underwent brain fluorodeoxyglucose positron emission tomography (FDG-PET) and neuropsychological assessments and completed PCS and psychiatric symptom rating scales. Compared to controls, Veterans with mTBI (with or without PTSD) exhibited decreased cerebral metabolic rate of glucose in the cerebellum, vermis, pons, and medial temporal lobe. They also exhibited subtle impairments in verbal fluency, cognitive processing speed, attention, and working memory, similar to those reported in the literature for patients with cerebellar lesions. These FDGPET imaging findings suggest that regional brain hypometabolism may constitute a neurobiological substrate for chronic PCS in Iraq combat Veterans with repetitive blast-trauma mTBI. Given the potential public health implications of these findings, further investigation of brain function in these Veterans appears warranted.
\end{abstract}

\section{Keywords}

Positron emission tomography; mild traumatic brain injury; post-concussive symptoms; cerebellum; blast; Veterans; cognition

\footnotetext{
*Corresponding Author: Elaine R. Peskind, MD, Mail Code S-116-MIRECC, Veterans Affairs Puget Sound Health Care System, 1660 S Columbian Way, Seattle, WA 98108 USA, Voice: +1-206-277-6935, Fax: +1-206-764-4856, peskind@uw.edu.
} 


\section{INTRODUCTION}

There is intense controversy regarding the etiology, course, and treatment of persistent somatic, cognitive, and behavioral symptoms experienced by many military personnel and Veterans following exposure to blast concussion. (Connors et al., 2009; Eibner et al., 2009; Hoge et al., 2009; Sigford et al., 2009) It is universally accepted that blast exposed personnel frequently meet American College of Rehabilitation Medicine (ACRM) criteria for acute mild traumatic brain injury (mTBI). (Kay et al., 1993) But the term "mTBI" increasingly has been applied to a very different chronic post-concussive syndrome of subtle cognitive deficits, headache, tinnitus, sleep disruption, daytime fatigue, irritability and other symptoms that persists for months and often years after the blast-induced acute mTBI. Whether these chronic symptoms reflect persistent structural or functional brain damage is not clear. An epidemiologic study in military personnel found that chronic post-concussive symptoms (except for headache) were more correlated with posttraumatic stress disorder (PTSD) and depression. (Schneiderman et al., 2008) These data would argue against persistent brain damage providing a neurobiologic basis for blast-related chronic post-concussive symptoms. On the other hand, many clinicians are convinced that war combatants' chronic postconcussive symptoms suggest real albeit subtle persistent brain damage. (Connors et al., 2009; Eibner et al., 2009; Ruff et al., 2008; Schneiderman et al., 2008; Sigford et al., 2009)

Demonstrating the presence of regional brain hypometabolism by fluorodeoxyglucose positron emission tomography (FDG PET) in combat Veterans with chronic post-concussive symptoms years after their last blast exposure mTBI, compared to a control group, would inform this debate. Here we report consistent regional hypometabolism in infratentorial and medial temporal brain regions in a sample of Iraq war combat Veterans with multiple episodes of blast exposure mTBI and persistent post-concussive symptoms.

\section{MATERIALS AND METHODS}

\section{Participants}

The Veterans Affairs Puget Sound Health Care System (VAPSHCS) and University of Washington (UW) institutional review boards approved all procedures and all the participants provided written informed consent before enrollment into the study. The mTBI Veteran participants were a convenience sample of 13 male Veteran warriors recruited from the VAPSHCS, all of whom had documented hazardous duty experience in Iraq with the US Armed Forces and had reported experiencing at least one blast exposure in the war zone that resulted in acute mTBI as defined by ACRM criteria, (Kay et al., 1993) except that a Glasgow Coma Scale (GCS) score, after 30 minutes, of 13-15, was not required, as GCS scores were not available in the combat setting. Control participants were 12 cognitively normal community volunteers (7 male, 5 female) recruited from the UW Alzheimer's Disease Research Center (ADRC), all of whom were medically healthy and had MiniMental State Examination scores of $29.4 \pm 1.0$ (mean \pm standard deviation [SD]) [range 2730]; Clinical Dementia Rating scores of zero; no evidence or history of cognitive (assessed with the ADRC Uniform Data Set [Morris et al., 2006]) or functional decline; and no history of blast exposure or head injury. For control participants older than 50 years, scores on delayed recall were greater than cutoff values set at one SD below the age-adjusted means for the Wechsler Logical Memory and New York University paragraph recall tests to ruleout mild memory impairment. None of the mTBI Veteran or control participants had a history of head injury with loss of consciousness (LOC) $\geq 30$ minutes; penetrating head wound; seizure disorder; insulin-dependent diabetes; current or past DSM-IV diagnoses of schizophrenia, other psychotic disorders, bipolar disorder, or dementia; or a DSM-IV diagnosis within the previous three months of alcohol or other substance abuse. Participants using medications likely to affect brain function, such as opioids, benzodiazepines or 
sedating antihistamines, were asked not to take those medications for 24 hours prior to PET imaging and neuropsychological testing.

\section{Blast-exposure Assessments}

Blast exposure and mTBI histories were obtained from mTBI Veteran participants during a clinical interview in which specific inquiries were made regarding total number of blast exposures accompanied by acute symptoms of TBI and/or LOC (both in Iraq and in other military and non-military settings) and history of non-blast exposure head injuries accompanied by acute symptoms of TBI and/or LOC (e.g., sports or motor vehicle accidentrelated concussion) prior to, during, and following military service.

\section{PET Imaging and Image Analysis}

Following intravenous infusion of $8-10 \mathrm{mCi}$ of $\left[{ }^{18} \mathrm{~F}\right]-\mathrm{FDG}$, each subject underwent a standard brain PET imaging protocol (20 minute emission scan and 25 minute Germanium-68 transmission scan for attenuation correction) in a GE advance scanner (GE Medical Systems, Milwaukee, Wisconsin), with in-plane and axial resolutions of $5 \mathrm{~mm}$ and $3 \mathrm{~mm}$ (full-width-half-maximum [FWHM]), respectively. Automated image co-registration, voxel intensity normalization to global brain activity, linear scaling/non-linear warping to Talairach stereotactic coordinate space, (Talairach and Tournoux, 1988) smoothing (3dimensional Gaussian kernel; $2.25 \mathrm{~mm} \mathrm{SD}$ ), and 3-dimensional stereotactic surface projection (3D-SSP) were performed using NEUROSTAT software (UW, Seattle, WA). (Minoshima et al., 1994a; Minoshima et al., 1994b) Voxelwise analyses of cerebral metabolic rate of glucose (CMRglu) differences between mTBI Veteran and control participants were performed by means of two-sample t-tests, with statistical significance evaluated (after transformation to Z-scores) using a random fields approach to control the Type I error rate approximately at $P=0.05(Z=3.5)$ for multiple comparisons. (Worsley et al., 1996) CMRglu in mTBI Veteran vs. control participants was also compared in stereotactically-defined volume-of-interest (VOI) analyses for the combined right and left frontal, temporal, parietal, occipital, anterior cingulate, posterior cingulate, medial frontal, medial parietal, medial temporal, insular, sensorimotor, parahippocampal, and visual cortices, thalami, caudate nuclei, amygdalae, cerebellar hemispheres, the cerebellar vermis, and the pons, by means of two-sample t-tests and a Bonferroni-corrected significance level of 0.003 to control for multiple comparisons in the 19 brain regions.

\section{Apolipoprotein E (APOE) Genotyping}

Genotypes were determined using previously described PCR conditions (Emi et al., 1988) and the HhaI restriction digest method. (Hixson and Vernier, 1990)

\section{Neuropsychological Testing}

All mTBI Veteran participants completed a battery of neuropsychological tests, including the Trail Making Test, (Reitan, 1958) Wechsler Adult Intelligence Scale-Revised (WAIS-R) Digit Symbol Substitution Test, (Wechsler, 1986) Ruff 2 and 7 Selective Attention Test, (Ruff et al., 1992) Controlled Oral Word Association Test (COWAT), (Ruff et al., 1996) Sentence Repetition Test, (Meyers et al., 2000) Wechsler Memory Scale-Revised Digit Span Forward and Backward, (Wechsler, 1987) and Wechsler Test of Adult Reading, (Psychological-Corporation, 2001) as well as the Computerized Neurocognitive BatteryPenn (CNP). (Gur et al., 2001) The latter assessed speed and accuracy of performance in a number of cognitive domains, including Abstraction and Mental Flexibility; Attention; Verbal Memory; Working Memory; Face Memory; Spatial Memory; Spatial Ability; and Sensory-Motor. The Test of Memory Malingering (TOMM) (Rees et al., 1998; Tombaugh, 1996) was used to assess level of effort during testing. 


\section{Behavioral Assessments}

Psychiatric diagnoses were established by means of a diagnostic interview using the Structured Clinical Interview for DSM-IV (SCID-IV). (First et al., 2002) PTSD symptoms were evaluated using the Clinician Administered PTSD Scale (CAPS) (Blake et al., 1995) and the PTSD Checklist-Military version (PCL-M); (Forbes et al., 2001) presence and severity of depressive symptoms with the Patient Health Questionnaire-9 (PHQ-9); (Kroenke et al., 2001) sleep disturbance with the Pittsburgh Sleep Quality Index (PSQI); (Buysse et al., 1989) and alcohol use with the Alcohol Use Disorders Identification TestConsumption Questions (AUDIT-C). (Bush et al., 1998) Quantification of current postconcussive symptoms was performed using the Neurobehavioral Symptom Inventory (NSI). (Cicerone and Kalmar, 1995)

\section{Neurological Assessments}

All subjects underwent a full neurological examination, including the Unified Parkinson's Disease Rating Scale (UPDRS) motor section. (Martinez-Martin et al., 1994) Olfactory function was assessed using the Brief Smell Identification Test. (Doty et al., 1996)

\section{RESULTS}

\section{Demographic characteristics}

One mTBI Veteran participant was dropped from the study due to TOMM scores suggesting poor effort. His scores and imaging findings are not included in the results presented here. The remaining $12 \mathrm{mTBI}$ Veteran participants were $32.0 \pm 8.5$ years of age (range 24-49 years) at the time of study enrollment. They had $13.8 \pm 1.9$ years of education (range 11-16 years). The cognitively normal community volunteer controls were $53.0 \pm 4.6$ years of age (range 49-56 years) and had $15.5 \pm 2.0$ years of education (range 12-19 years).

\section{APOE*4 allele status}

One mTBI Veteran and 4 control participants were $A P O E^{*} 4$ allele carriers.

\section{Blast Exposure Characteristics}

The mTBI Veteran participants had experienced $13 \pm 14$ (range 3-51) blast exposure mTBI episodes while deployed to Iraq (of which $1.0 \pm 1.35$ [range $0-4$ ] were accompanied by LOC) and $30 \pm 36$ (range 5-102) blast exposure mTBI episodes in their military careers (of which all accompanied by LOC occurred in Iraq). Mean time between mTBI Veteran participants' most recent blast exposure and study participation was $3.5 \pm 1.2$ years (range 2-5 years). Lifetime episodes of head trauma with LOC were $1.75 \pm 1.76$ (range $0-5$ ), indicating that nearly all LOC episodes in the mTBI Veteran participants were associated with military service.

\section{Brain FDG-PET Imaging}

Overall, the mTBI Veteran participants exhibited a consistent pattern of decreased CMRglu in infratentorial structures (i.e., cerebellum, vermis, and pons) as well as medial temporal cortex compared to the controls. Z-scores maps of glucose hypometabolism (i.e., relative to the control group of community volunteers) for each of the $12 \mathrm{mTBI}$ Veteran participants are presented in Figure 1. A composite Z-score map of glucose hypometabolism (again, relative to the control group) based on the mean CMRglu values for the mTBI Veteran group is presented in Figure 2. The locations of voxels for which CMRglu values were significantly lower in mTBI Veterans vs. controls are presented in Table 1. These brain structures included the left cerebellar pyramis and uvula, the right cerebellar inferior semilunar lobule, the middle temporal (Brodmann Areas 21 and 37) and fusiform gyri, and the 
cuneus. Similar, albeit not identical, findings were obtained in VOI analyses, which demonstrated relative hypometabolism in mTBI Veteran vs. control participants in the combined right and left medial temporal lobe $(0.60 \pm 0.03$ vs. $0.63 \pm 0.04, \mathrm{p}<0.05)$ and cerebellar hemispheres $(0.83 \pm 0.04$ vs. $0.90 \pm 0.05$, $\mathrm{p}<0.001)$, as well as the cerebellar vermis ( $0.89 \pm 0.02$ vs. $0.92 \pm 0.03, \mathrm{p}<0.05)$, and the pons $(0.65 \pm 0.04$ vs. $0.70 \pm 0.04, \mathrm{p}<0.01)$, while thalamic CMRglu values were only marginally lower $(1.00 \pm 0.07$ vs. $1.06 \pm 0.09, \mathrm{p}=0.06)$. However, only the cerebellar hypometabolism remained significant after Bonferroni correction. Infratentorial and medial temporal hypometabolism appeared to be unrelated to PTSD status, as similar patterns of regional hypometabolism were exhibited by mTBI Veteran participants one and ten, who had CAPS scores of five and zero, respectively.

\section{Neuropsychological Test Performance}

Neuropsychological test scores for the mTBI Veteran participants were largely within normal limits, with some areas of inefficiency. Specifically, mean scores on attention and processing speed measures (i.e., Sentence Repetition, Ruff $2 \& 7$ speed, and Category fluency and COWAT) were 0.5-1.0 SD below age- and education-matched norms. On the computerized test battery, mTBI Veteran participants demonstrated low average mean accuracy scores for CNP Working Memory $(\mathrm{Z}=-0.82 \pm 2.52)$ relative to healthy controls. Their mean response times were low average on CNP verbal memory measures $(Z=-0.81$ $\pm 0.84)$ and even slower on CNP measures of spatial ability $(Z=-1.7 \pm 1.25)$. In contrast, mean response times on $\mathrm{CNP}$ attention measures were above average $(\mathrm{Z}=0.80 \pm 0.67)$.

\section{Psychiatric Diagnoses and Symptom Ratings}

Ten of the 12 mTBI Veteran participants met DSM-IV criteria for PTSD by SCID-IV criteria and the CAPS. Of the 2 subjects without PTSD, CAPS scores were five and zero, respectively. Three mTBI Veteran participants met criteria for current major depression, one for generalized anxiety disorder, and one for panic disorder. The mTBI Veteran participant's mean scores were $12.9 \pm 1.8$ (range 9-15) on the Combat Experiences Scale; $55.7 \pm 30.8$ on the CAPS; $32.3 \pm 18.0$ on the PCL-M; $9.7 \pm 8.5$ on the PHQ-9; $9.1 \pm 3.1$ on the PSQI; and $5.6 \pm 2.5$ on the AUDIT-C.

\section{Post-concussive Symptoms}

NSI items endorsed as moderate or severe in intensity by the mTBI Veteran participants included: ringing in ears (83\%); forgetfulness (75\%); feeling anxious or tense $(75 \%)$; difficulty falling or staying asleep (67\%); irritability (67\%); sensitivity to noise (67\%); hearing difficulty (67\%); poor frustration tolerance (67\%); mood swings (67\%); headaches (67\%); feeling depressed or sad (67\%); slowed thinking (67\%); poor concentration (67\%); difficulty making decisions (42\%); sensitivity to light (33\%); fatigue (33\%); poor coordination (33\%); numbness on parts of body (33\%); loss of balance (25\%); vision problems (25\%); getting into fights (25\%); apathy (25\%); disinhibition (17\%); feeling dizzy (17\%); change in taste/smell (8\%); slowness in speech (8\%); nausea (8\%); and loss/ increased appetite (8\%).

\section{Concurrent medications}

Medications with potential central nervous system effects being taken by mTBI Veteran participants included: oxycodone $5 \mathrm{mg} 4 \mathrm{x} /$ day $(1 / 12)$, prazosin $(5 / 12)$, selective serotonin reuptake inhibitors $(2 / 12)$, serotonin and norepinephrine reuptake inhibitors $(3 / 12)$, mirtazapine (2/12), benzodiazepines (1/12), and atypical antipsychotics (1/12). Control participants were taking no medications except for one taking trazodone for insomnia. 


\section{Neurologic Findings}

None of the mTBI Veteran participants reported focal neurological symptoms and none exhibited focal deficits on neurologic examination. UPDRS ratings were zero in 10/12 mTBI Veteran participants; the other two participants had ratings of two and seven, respectively. Brief Smell Identification Test scores were 12/12 in four mTBI Veterans; 11/12 in six; and $10 / 12$ in two, suggesting intact olfactory function in these participants.

\section{DISCUSSION}

This is the first report of FDG-PET in military blast-exposure mTBI. We demonstrated consistent regional hypometabolism in infratentorial (cerebellum, vermis, and pons) and medial temporal brain regions in Iraq combat Veterans with multiple episodes of blastrelated mTBI. These participants also exhibited subtle impairments in complex information processing, with mild reductions in verbal fluency, processing speed, and aspects of attention and working memory. These findings suggest the possibility that persistent somatic, cognitive, and behavioral symptoms reported by some Iraq war Veterans with repetitive blast-related mTBI may have a neurobiological substrate that can be objectively measured by means of brain FDG-PET imaging, and that persistent post-concussive symptoms in Iraq war Veterans with repetitive blast exposure mTBI should not be solely attributed to comorbid psychiatric disorders until the possible contribution of occult brain damage has been fully evaluated in replication studies.

Our mTBI Veteran participants' regional distribution of glucose hypometabolism (i.e., cerebellum, vermis, pons, and medial temporal lobe), cognitive domain deficits (i.e., processing speed, attention, working memory, and verbal fluency), and behavioral symptoms (i.e., irritability, poor frustration tolerance, mood swings, getting into fights, and disinhibition) recapitulate some, albeit not all, components of the "cerebellar cognitive affective syndrome," which has been identified in patients with isolated cerebellar pathology. (Schmahmann, 2004; Stoodley and Schmahmann, 2009) Particularly striking is the close correspondence between the cerebellar and subcortical brain structures exhibiting relative hypometabolism in our mTBI Veteran participants and the neuroanatomy of corticocerebellar-cortical circuits, in which afferent cortical projections converge in the pons and pass through the cerebellar peduncles to the cerebellar hemispheres and vermis, from which efferent projections converge in the deep cerebellar nuclei and traverse the peduncles back to the thalamus and thence to motor and association cortices. (Schmahmann, 2004) Consistent with the findings in our mTBI Veteran participants, Warden et al (Warden et al., 2009) described a single Iraq war soldier with persistent post-concussive symptoms after blast-exposure mTBI that was associated with transient isolated left cerebellar axonal injury on magnetic resonance diffusion tensor imaging. That cerebellar injury may play a role in the cognitive and behavioral symptoms exhibited by our mTBI Veteran participants is supported by recent neuropsychological and functional imaging findings implicating posterior cerebellar cortex lesions with executive, visual spatial, and linguistic impairments and vermal lesions with affective dysregulation. (Stoodley and Schmahmann, 2009) In addition, cerebellar dysfunction has recently been implicated in the pathogenesis of similar symptoms in civilian mTBI patients. (Hattori et al., 2009; Kato et al., 2007; Park et al., 2007a)

The mechanisms by which blast exposures associated with acute episodes of mTBI may result in metabolic abnormalities in the infratentorial and medial temporal regions, as exhibited by our mTBI Veteran subjects, remain obscure. That these brain regions may be selectively vulnerable to damage in response to blast or blunt-force impacts associated with acute $\mathrm{mTBI}$ is supported by finite element brain models demonstrating that strain forces developing in the temporal lobe, corpus callosum, fornix, and midbrain during player 
impacts in football are associated with concussive symptoms, (Viano et al., 2005) that explosive blast waves produce elevated shear forces in midbrain/pontine, cerebellar, and medial temporal brain regions, even when head movement is negligible, (Taylor and Ford, 2009) and that blast exposure in experimental animals is associated with cerebellar lesions. (Saljo et al., 2008) In addition, cerebellar Purkinje neurons are particularly vulnerable to mechanical trauma, even when applied to the cerebrum, rather than to the cerebellum itself. (Park et al., 2007b)

One limitation of our study is that the mean age of the control group is approximately 21 years older than the mTBI Veteran group. However, age effects on resting brain FDG-PET are minimal between young and middle-aged subjects and age-related changes observed in elderly subjects manifest predominantly in frontal cortical regions, (Willis et al., 2002) making it unlikely that the regional hypometabolism pattern seen in our mTBI Veteran participants is an artifact of their younger age. Because our control participants were not military personnel, it may be argued that unmeasured premorbid and comorbid factors related to military service may have confounded our FDG-PET imaging findings. (McLean et al., 1993) However, absent evidence that CMRglu abnormalities are highly prevalent in Iraq war Veterans without blast exposure, it is unlikely that such factors account for our findings. Another limitation of our study is that 10 of our $12 \mathrm{mTBI}$ Veteran participants also met diagnostic criteria for PTSD, potentially confounding psychiatric diagnosis and blast exposure effects on regional CMRglu. However, extant data demonstrating resting FDGPET abnormalities in PTSD patients vs. subjects without PTSD are limited and conflicting. Bremner et al, (Bremner et al., 1997) reported relative temporal lobe resting hypometabolism in 10 Vietnam combat Veterans with PTSD (by DSM-III criteria) compared to 10 community volunteers, but no difference in resting thalamic, pontine, midbrain, cerebellar, or global brain CMRglu. In contrast, Molina et al, (Molina et al., 2007) reported elevated resting temporal lobe and cerebellar and normal thalamic CMRglu in combat Veterans with vs. without PTSD. These findings suggest that confounding effects of comorbid PTSD are unlikely to account for the regional hypometabolism seen in our mTBI Veteran participants. However, FDG-PET imaging of Iraq war Veterans with blast-related mTBI who do not have PTSD will be required to definitively answer this question.

These findings suggest that chronic post-concussive symptoms in Iraq war combat Veterans with repetitive blast-related acute mTBI may reflect persistent dysfunction in infratentorial and medial temporal brain regions that can be objectively interrogated by means of brain FDG-PET imaging. Given the potential implications of these findings for the evaluation and treatment of blast-exposed Iraq war military personnel and Veterans, replication in larger samples is urgently needed. The ongoing debate over the etiology of chronic postconcussive symptoms in Iraq war Veterans should not be considered resolved until it can be informed by the results of such studies.

\section{Acknowledgments}

This material is based upon work supported, in part, by the Northwest Network Mental Illness, Research, Education, and Clinical Center (MIRECC) and Office of Research and Development Medical Research Service, Department of Veterans Affairs; by grants P50 AG05136, K08 AG023670, and R01 NS045254 from the National Institutes of Health; and by a grant from an anonymous foundation.

\section{References}

Blake DD, Weathers FW, Nagy LM, Kaloupek DG, Gusman FD, Charney DS, Keane TM. The development of a Clinician-Administered PTSD Scale. J Trauma Stress. 1995; 8:75-90. [PubMed: 7712061] 
Bremner JD, Innis RB, Ng CK, Staib LH, Salomon RM, Bronen RA, Duncan J, Southwick SM, Krystal JH, Rich D, Zubal G, Dey H, Soufer R, Charney DS. Positron emission tomography measurement of cerebral metabolic correlates of yohimbine administration in combat-related posttraumatic stress disorder. Arch Gen Psychiatry. 1997; 54:246-254. [PubMed: 9075465]

Bush K, Kivlahan DR, McDonell MB, Fihn SD, Bradley KA. The AUDIT alcohol consumption questions (AUDIT-C): an effective brief screening test for problem drinking. Ambulatory Care Quality Improvement Project (ACQUIP). Alcohol Use Disorders Identification Test. Arch Intern Med. 1998; 158:1789-1795. [PubMed: 9738608]

Buysse DJ, Reynolds CF 3rd, Monk TH, Berman SR, Kupfer DJ. The Pittsburgh Sleep Quality Index: a new instrument for psychiatric practice and research. Psychiatry Res. 1989; 28:193-213.

[PubMed: 2748771]

Cicerone K, Kalmar K. Persistent postconcussion syndrome: the structure of subjective complaints after mild traumatic brain injury. J Head Trauma Rehabil. 1995; 10:1-17.

Connors S, Gordon WA, Hovda DA. Care of war veterans with mild traumatic brain injury. N Engl J Med. 2009; 361:536-537. author reply 537-538. [PubMed: 19645084]

Doty RL, Marcus A, Lee WW. Development of the 12-item Cross-Cultural Smell Identification Test (CC-SIT). Laryngoscope. 1996; 106:353-356. [PubMed: 8614203]

Eibner C, Schell TL, Jaycox LH. Care of war veterans with mild traumatic brain injury. N Engl J Med. 2009; 361:537. author reply 537-538. [PubMed: 19645085]

Emi M, Wu LL, Robertson MA, Myers RL, Hegele RA, Williams RR, White R, Lalouel JM. Genotyping and sequence analysis of apolipoprotein E isoforms. Genomics. 1988; 3:373-379. [PubMed: 3243553]

First, MB.; Spitzer, RL.; Gibbon, M.; Williams, JBW. Structured Clinical Interview for DSM-IV-TR Axis I Disorders, Research Version, Patient Edition. (SCID-I/P). Biometrics Research, New York State Psychiatric Institute; New York: 2002.

Forbes D, Creamer M, Biddle D. The validity of the PTSD checklist as a measure of symptomatic change in combat-related PTSD. Behav Res Ther. 2001; 39:977-986. [PubMed: 11480838]

Gur RC, Ragland JD, Moberg PJ, Turner TH, Bilker WB, Kohler C, Siegel SJ, Gur RE. Computerized neurocognitive scanning: I. Methodology and validation in healthy people. Neuropsychopharmacology. 2001; 25:766-776. [PubMed: 11682260]

Hattori N, Swan M, Stobbe GA, Uomoto JM, Minoshima S, Djang D, Krishnananthan R, Lewis DH. Differential SPECT activation patterns associated with PASAT performance may indicate frontocerebellar functional dissociation in chronic mild traumatic brain injury. J Nucl Med. 2009; 50:1054-1061. [PubMed: 19525460]

Hixson JE, Vernier DT. Restriction isotyping of human apolipoprotein E by gene amplification and cleavage with HhaI. J Lipid Res. 1990; 31:545-548. [PubMed: 2341813]

Hoge CW, Goldberg HM, Castro CA. Care of war veterans with mild traumatic brain injury--flawed perspectives. N Engl J Med. 2009; 360:1588-1591. [PubMed: 19369664]

Kato T, Nakayama N, Yasokawa Y, Okumura A, Shinoda J, Iwama T. Statistical image analysis of cerebral glucose metabolism in patients with cognitive impairment following diffuse traumatic brain injury. J Neurotrauma. 2007; 24:919-926. [PubMed: 17600509]

Kay T, Harrington DE, Adams R, Anderson T, Berrol S, Cicerone K, Dahlberg C, Gerber D, Goka R, Harley P, Hilt J, Horn L, Lehmkuhl D, Malec J. Mild Traumatic Brain Injury Committee of the Head Injury Interdisciplinary Special Interest Group of the American Congress of Rehabilitation Medicine. Definition of mild traumatic brain injury. J Head Trauma Rehabil. 1993; 8:86-87.

Kroenke K, Spitzer RL, Williams JB. The PHQ-9: validity of a brief depression severity measure. J Gen Intern Med. 2001; 16:606-613. [PubMed: 11556941]

Martinez-Martin P, Gil-Nagel A, Gracia LM, Gomez JB, Martinez-Sarries J, Bermejo F. Unified Parkinson's Disease Rating Scale characteristics and structure. The Cooperative Multicentric Group. Mov Disord. 1994; 9:76-83. [PubMed: 8139608]

McLean A Jr, Dikmen SS, Temkin NR. Psychosocial recovery after head injury. Arch Phys Med Rehabil. 1993; 74:1041-1046. [PubMed: 8215854]

Meyers JE, Volkert K, Diep A. Sentence repetition test: updated norms and clinical utility. Appl Neuropsychol. 2000; 7:154-159. [PubMed: 11125709] 
Minoshima S, Koeppe RA, Frey KA, Ishihara M, Kuhl DE. Stereotactic PET atlas of the human brain: aid for visual interpretation of functional brain images. J Nucl Med. 1994a; 35:949-954. [PubMed: 8195881]

Minoshima S, Koeppe RA, Frey KA, Kuhl DE. Anatomic standardization: linear scaling and nonlinear warping of functional brain images. J Nucl Med. 1994b; 35:1528-1537. [PubMed: 8071705]

Molina ME, Isoardi R, Prado MN, Bentolila S. Basal cerebral glucose distribution in long-term posttraumatic stress disorder. World J Biol Psychiatry. 2007:1-9.

Morris JC, Weintraub S, Chui HC, Cummings J, Decarli C, Ferris S, Foster NL, Galasko D, GraffRadford N, Peskind ER, Beekly D, Ramos EM, Kukull WA. The Uniform Data Set (UDS): clinical and cognitive variables and descriptive data from Alzheimer Disease Centers. Alzheimer Dis Assoc Disord. 2006; 20:210-216. [PubMed: 17132964]

Park E, Ai J, Baker AJ. Cerebellar injury: clinical relevance and potential in traumatic brain injury research. Prog Brain Res. 2007a; 161:327-338. [PubMed: 17618988]

Park E, Liu E, Shek M, Park A, Baker AJ. Heavy neurofilament accumulation and alpha-spectrin degradation accompany cerebellar white matter functional deficits following forebrain fluid percussion injury. Exp Neurol. 2007b; 204:49-57. [PubMed: 17070521]

Psychological-Corporation. Wechsler Test of Adult Reading. Psychological Corporation; San Antonio, TX: 2001.

Rees LM, Tombaugh TN, Gansler DA, Moczynski NP. Five validation experiments of the Test of Memory Malingering (TOMM). Psychological Assessment. 1998; 10:10-20.

Reitan RM. Validity of the Trail-Making Test as an indication of organic brain damage. Perceptual and Motor Skills. 1958; 8:271-276.

Ruff RL, Ruff SS, Wang XF. Headaches among Operation Iraqi Freedom/Operation Enduring Freedom veterans with mild traumatic brain injury associated with exposures to explosions. J Rehabil Res Dev. 2008; 45:941-952. [PubMed: 19165684]

Ruff RM, Light RH, Parker SB, Levin HS. Benton Controlled Oral Word Association Test: reliability and updated norms. Arch Clin Neuropsychol. 1996; 11:329-338. [PubMed: 14588937]

Ruff RM, Niemann H, Allen CC, Farrow CE, Wylie T. The Ruff 2 and 7 Selective Attention Test: a neuropsychological application. Percept Mot Skills. 1992; 75:1311-1319. [PubMed: 1484803]

Saljo A, Arrhen F, Bolouri H, Mayorga M, Hamberger A. Neuropathology and pressure in the pig brain resulting from low-impulse noise exposure. J Neurotrauma. 2008; 25:1397-1406. [PubMed: 19146459]

Schmahmann JD. Disorders of the cerebellum: ataxia, dysmetria of thought, and the cerebellar cognitive affective syndrome. J Neuropsychiatry Clin Neurosci. 2004; 16:367-378. [PubMed: 15377747]

Schneiderman AI, Braver ER, Kang HK. Understanding sequelae of injury mechanisms and mild traumatic brain injury incurred during the conflicts in Iraq and Afghanistan: persistent postconcussive symptoms and posttraumatic stress disorder. Am J Epidemiol. 2008; 167:14461452. [PubMed: 18424429]

Sigford B, Cifu DX, Vanderploeg R. Care of war veterans with mild traumatic brain injury. N Engl J Med. 2009; 361:536. author reply 537-538. [PubMed: 19641217]

Stoodley CJ, Schmahmann JD. Functional topography in the human cerebellum: a meta-analysis of neuroimaging studies. Neuroimage. 2009; 44:489-501. [PubMed: 18835452]

Talairach, J.; Tournoux, P. 3-Dimensional Proportional System: An Approach to Cerebral Imaging. Thieme Medical Publishers; New York: 1988. Co-planar Stereotaxic Atlas of the Human Brain.

Taylor PA, Ford CC. Simulation of blast-induced early-time intracranial wave physics leading to traumatic brain injury. J Biomech Eng. 2009; 131:061007. [PubMed: 19449961]

Tombaugh, TN. Test of Memory Malingering. Multi-Health Systems, Inc; North Tonawanda, NY: 1996.

Viano DC, Casson IR, Pellman EJ, Zhang L, King AI, Yang KH. Concussion in professional football: brain responses by finite element analysis: part 9. Neurosurgery. 2005; 57:891-916. discussion 891-916. [PubMed: 16284560] 
Warden DL, French LM, Shupenko L, Fargus J, Riedy G, Erickson ME, Jaffee MS, Moore DF. Case report of a soldier with primary blast brain injury. Neuroimage. 2009; 47(Suppl 2):T152-153. [PubMed: 19457364]

Wechsler, D. WAIS-R Administration and Scoring Manual. The Psychological Corporation; San Antonio, TX: 1986.

Wechsler, D. Wechsler Memory Scale-Revised. The Psychological Corporation; San Antonio, TX: 1987.

Willis MW, Ketter TA, Kimbrell TA, George MS, Herscovitch P, Danielson AL, Benson BE, Post RM. Age, sex and laterality effects on cerebral glucose metabolism in healthy adults. Psychiatry Res. 2002; 114:23-37. [PubMed: 11864807]

Worsley, KJ.; Marrett, S.; Neelin, P.; Evans, AC. A unified statistical approach for determining significant signals in location and scale space images of cerebral activation. In: Myers, R.; Cunningham, VJ.; Bailey, D.; Jones, T., editors. Quantification of brain function using PET. Academic Press; San Diego: 1996. p. 327-333. 


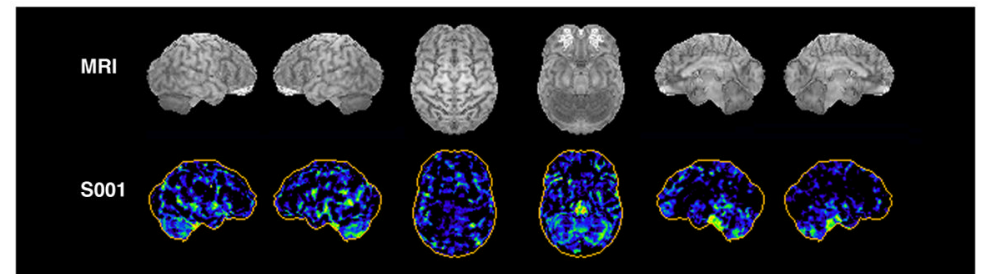

S002
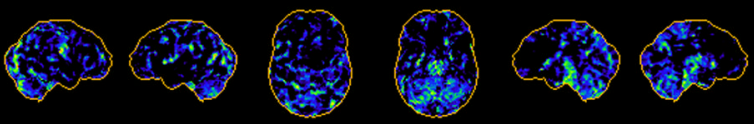

S003
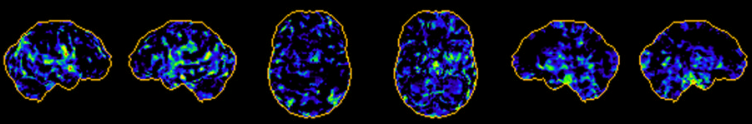

$\mathrm{SO04}$
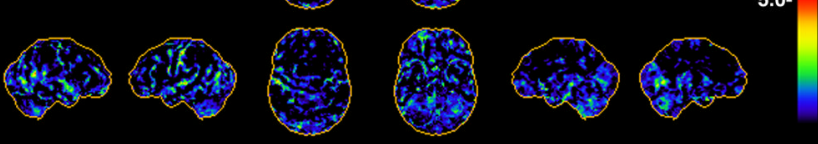

S005
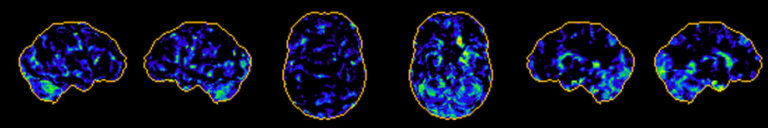

S006
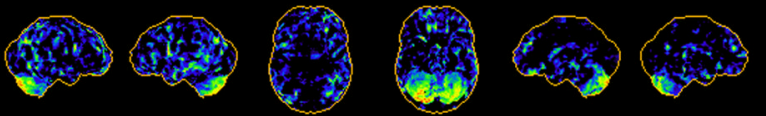

S007
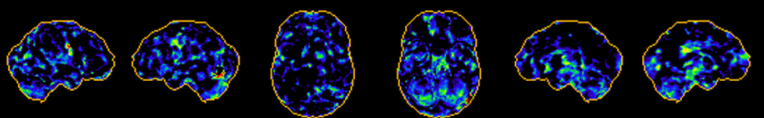

S008
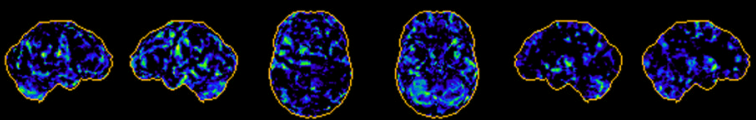

S009
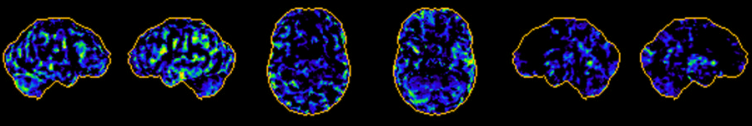

S010


S011
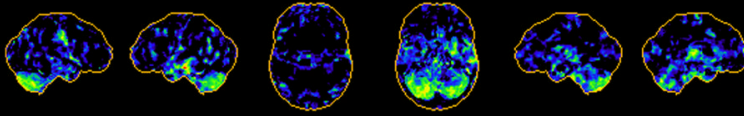

Z

S012
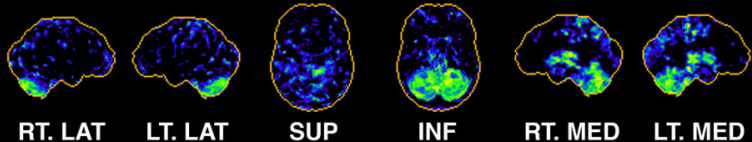

Figure 1.

Magnetic resonance imaging brain templates (top row) and voxel-wise Z-score maps for each mTBI Veteran participant (S001-S012, lower rows) showing patterns of hypometabolism relative to community volunteer control group $(n=12)$. Views are Right Lateral (RT LAT), Left Lateral (LT LAT), Right Medial (RT MED), Left Medial (LT MED), Superior (SUP), and Inferior (INF). Vertical bar shows image color vs. Z-score scale. 


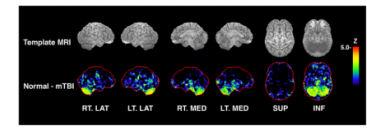

Figure 2.

Magnetic Resonance Imaging brain template (top row) and Z-score map of cerebral glucose metabolism difference between mTBI Veteran group $(n=12)$ and community volunteer control group $(\mathrm{n}=12)$ (bottom row). Views and vertical bar are the same as in Figure 1. 
Table 1

Locations of Voxels Exhibiting Significantly Lower CMRglu in mTBI Veteran vs. Control Participants

\begin{tabular}{|l|c|c|}
\hline Structure & Z-score & Stereotactic Coordinates (mm)* \\
\hline Left cerebellum, posterior lobe, pyramis & -4.0 & $(-33,-71,-34)$ \\
\hline Middle temporal gyrus, BA 21 & -4.0 & $(-60,-51,7)$ \\
\hline Left cerebellum, posterior lobe, uvula & -4.0 & $(-33,-73,-25)$ \\
\hline Middle temporal gyrus, BA 21 & -3.9 & $(60,-33,-11)$ \\
\hline Cuneus, BA 17 & -3.7 & $(6,-94,4)$ \\
\hline Fusiform gyrus, BA 20 & -3.7 & $(46,-26,-16)$ \\
\hline Right cerebellum, posterior lobe, inferior semi-lunar lobule & -3.6 & $(39,-62,-36)$ \\
\hline Middle temporal gyrus, BA 37 & -3.52 & $(51,-62,9)$ \\
\hline
\end{tabular}

Talairach and Tournoux atlas. (Talairach and Tournoux, 1988) For x-axis coordinates, negative values are right of midline.

CMRglu: cerebral glucose metabolic rate, mTBI: mild traumatic brain injury, BA: Brodmann Area 